\title{
SIMULATION OF FATUQUE CRACK PROPAGATION IN DUCTILE METALS BY BLUNTING AND RE-SHARPENING
}

\author{
Vladislav Levkovitch ${ }^{1}$, Rainer Sievert ${ }^{2}$, Bob Svendsen ${ }^{1}$ \\ ${ }^{1}$ Deprtment of Mechanical Engineering, University of Dortmund, D-44227 Dortmund, Germany \\ ${ }^{2}$ Federal Institute for Materials Research and Testing, D-12200 Berlin, Germany
}

\section{ABSTRACT}

Laird and Smith [1] proposed a plastic sliding-off mechanism for the stage II fatigue crack growth via striation formation. In their view, the fatigue crack extension results solely from the different character of the deformation at the crack tip during loading and unloading. In particular, the crack tip blunts during the loading stage and folds into a double notch during the unloading stage, resulting in striation formation. In order to verify Laird's plastic blunting mechanism for crack propagation via striation formation for ductile fcc single crystals, a FEM calculation was performed for a rectangular plate with an initially sharp crack under plane strain conditions. The plate was subjected to a fully reversed tension-to-pressure cyclic load perpendicular to the crack plane (Mode 1). No initial radius for the crack tip was assumed. The actual shape of the crack tip followed from an initially sharp crack by repeated re-meshing. To model the constitutive behavior typical for ductile fcc single crystals, a geometrically nonlinear form of Cailletaud's model based on the multiplicative elastic-plastic decomposition of the deformation gradient was implemented into the FE program ABAQUS. Only octahedral slip systems were considered. The crack propagation simulations were carried out for cracks with crack plane (001) for two different crack growth orientations [110] and [100]. The external cyclic load was imposed in the (001) direction. Using repeated re-meshing for severely distorted elements at the advancing crack tip, deformation patterns in the sense of Laird's mechanism for fatigue-crack propagation with striation formation were obtained for [110] crack growth direction. The simulation for [100] crack growth direction with the same stress level as for [110] direction also yielded crack extension by the progressive large deformations, but without striations formation.

\section{INTRODUCTION}

While the statement that a fatigue crack advances by an increment $\Delta a$ in each cycle, except at very low growth rates in the near-threshold region, is generally accepted, there is a lack of agreement as to which mechanism controls the crack growth process. Two different mechanisms have been proposed to describe fatigue crack extension. One is based on a plastic sliding-off at the tip of the advancing crack, while the other is in terms of damage accumulation at the crack tip. The slidingoff mechanism associates crack extension with blunting of the crack tip during loading followed by re-sharpening upon unloading. This approach, which is usually used to explain fatigue crack growth via a mechanism of striation formation, was fist proposed by Laird and Smith [1] for smooth blunting, and later by Neumann [2] and Pelloux [3] for blunting via alternating shear.

Fatigue striations are ripples on the fracture surface, and are often observed as a result of subcritical crack growth in ductile materials during cyclic loading. For cyclic loads in the Paris regime of fatigue crack advance, it has been found that the spacing between adjacent striations correlates with the experimentally measured average crack growth rate per cycle.

FEM simulations of the crack propagation via blunting and re-sharpening under cyclic loading have been described in Gu [4] and Tvergaard [5] for isotropic materials. These simulations were carried out for moderately large deformations at the crack tip in order to avoid the severe distortions of the elements at the crack tip. No striations were obtained in these papers. In order to simulate the striations formation much larger deformations at the crack tip need to be considered which is only possible by usage of a repeated re-meshing technique. 


\section{PLASTIC BLUNTING AND RE-SHARPENING MECHANISM DUE TO LAIRD}

In order to explain the crack growth via the striations formation, Laird [1] performed direct observations of the crack-tip geometry on some ductile metals corresponding to different stages of the stress cycle. The mechanism of crack propagation deduced from these observations is now called plastic blunting process or Laird's model and is shown in Figure 1. The initial zero load corresponds to a well developed Stage II crack with the fracture surface exhibiting striations (a). As the tensile load is applied, the metal yields plastically due to high stress concentration. This plastic deformation is highly concentrated in the slip zones along the planes of maximum shear stress (b). When the load is further increased, the slip zones at the tip broaden and the crack tip blunts to a semicircular configuration. The crack tip thus is effectively shifted (c). If the far-field stress is reversed, the crack tip re-sharpens by buckling and folding of the newly created surface into a double notch resulting in a striation formation (d ) and (e). Since the closure of the crack during compression cannot fully negate the blunting and the attendant extension of the crack during the preceding tension load, net crack growth occurs during a fatigue cycle, leading to the formation of a striation. Laird's model applies to a wide variety of ductile materials, including polymers, because of its general description of the plastic blunting process.

(a)

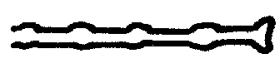

(b)

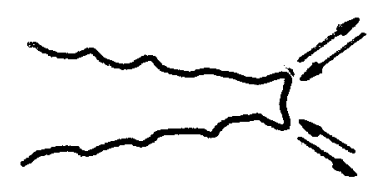

(c)

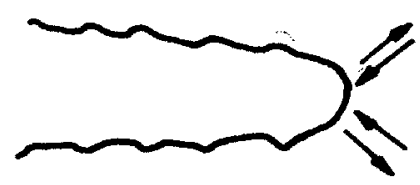

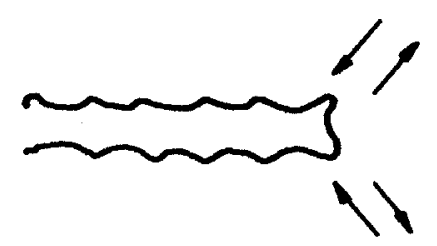

(d)

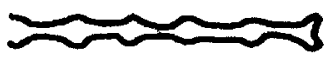

(e)

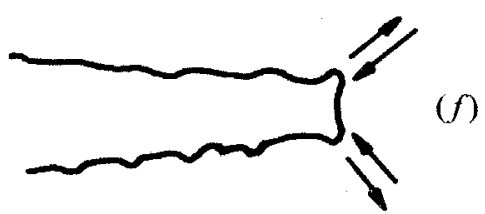

Figure 1: An idealization of plastic blunting and re-sharpening process leading to stage II fatigue crack growth in fully reversed fatigue (after Laird).

\section{FEM MODELLING}

In order to verify Laird's plastic blunting mechanism for crack propagation via striation formation for ductile fcc single crystals, a FEM calculation was performed for a rectangular plate with an initially sharp crack under plane strain conditions. The plate was subjected to a fully reversed tension-to-pressure cyclic load perpendicular to the crack plane (Mode 1). For symmetry reasons only one half plate needs to be considered. No initial radius for the crack tip was assumed. The actual shape of the crack tip follows from the initially sharp crack by repeated re-meshing. Due to the cyclic nature of the plastic deformation at the crack tip, a plasticity model with kinematic hardening is required. A geometrically nonlinear version of Cailletaud's model (e.g., Méric [6]) based on the elastic-plastic decomposition of the deformation $\boldsymbol{F}=\boldsymbol{F}_{e} \boldsymbol{F}_{p}$ was implemented into the FE program ABAQUS. To this end, only octahedral slip systems were considered. The crack 
propagation simulations were carried out for cracks with crack plane (001) for crack growth in the directions [110] and [100]. The external cyclic load was imposed in (001) direction.

In single crystals, inelastic deformation results from slip process due to the dislocation motion on crystallographic planes, resulting in an evolution of Fp of the form

$$
\dot{F}_{P}=\sum_{\alpha} \dot{\gamma}_{\alpha} \boldsymbol{s}_{\alpha} \circ \boldsymbol{n}_{\alpha} \dot{F}_{P}
$$

where $\boldsymbol{s}_{\alpha}$ and $\boldsymbol{n}_{\alpha}$ are the slip direction and the normal vector to the slip plane of the slip system $\alpha$, and gamma is the glide-system slip. The flow rule is given by

$$
\dot{\gamma}_{\alpha}=\left\langle\frac{\left|\tau_{\alpha}-x_{\alpha}\right|-r_{0}}{k}\right\rangle^{n} \operatorname{sign}\left(\tau_{\alpha}-x_{\alpha}\right) \text {, }
$$

with the resolved shear stress $\tau_{\alpha}$ on the slip system $\alpha$ and the evolution equations for kinematic hardening:

$\dot{x}_{\alpha}=c\left(a \dot{\gamma}_{\alpha}-x_{\alpha}\left|\dot{\gamma}_{\alpha}\right|\right)$

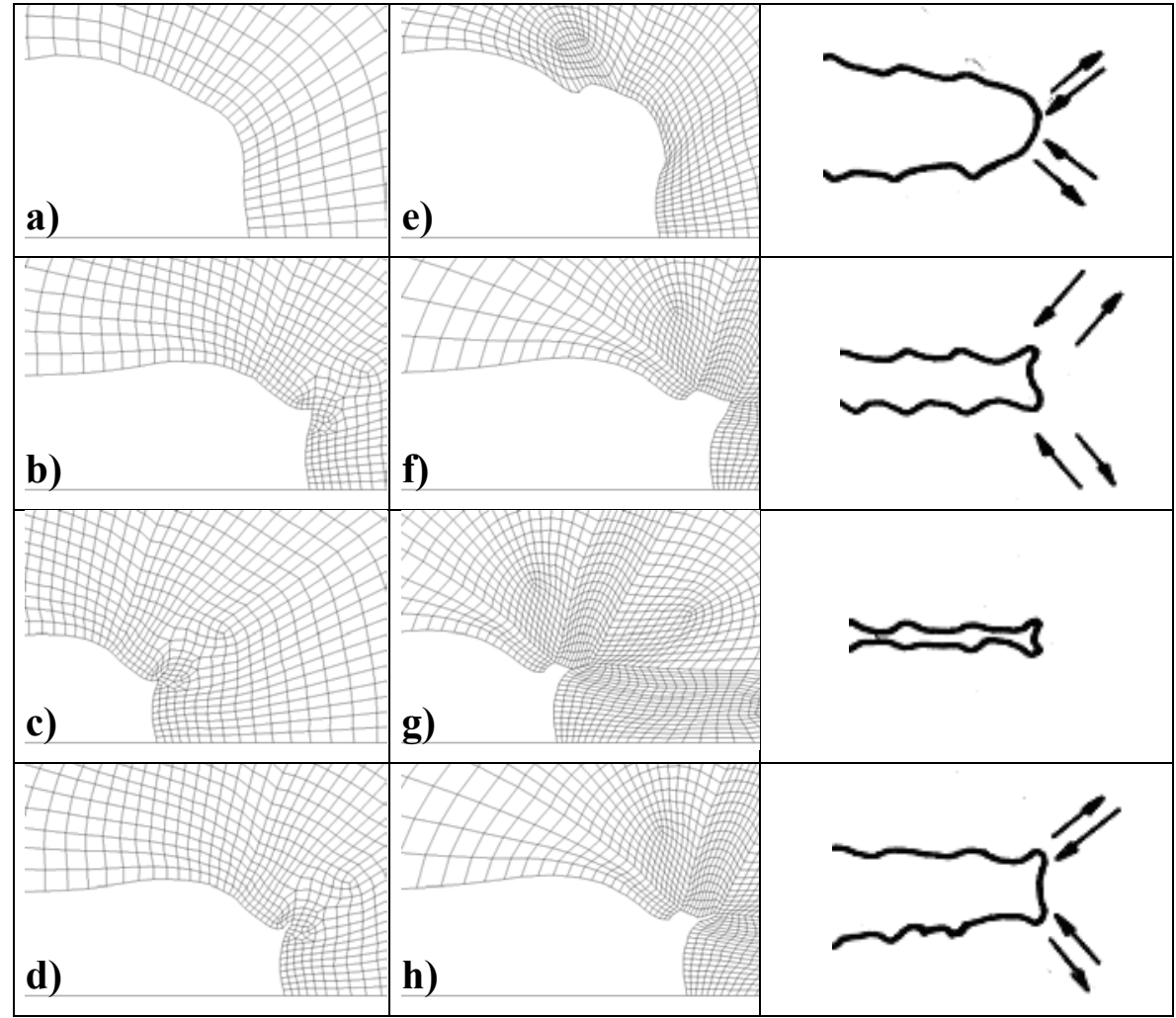

Figure 2: Simulation of crack propagation by blunting and re-sharpening for crack plane orientation (001) and crack growth direction [110]: first row - peak tensile load, second row - zero load, third row - peak compressive load, forth row - zero load. 
Figure 2 shows the deformation evolution at the crack tip for the crack orientation (001)[110]. Starting from an initially sharp crack, as the tensile load increases the crack blunts to a semicircular form (a). Due to the dissimilarity of the deformation in the unloading stage the crack tip begins to buckle (b) and folds up in the compressive stage (c). In order to prevent the material from the mutual penetration in the folding area self contact has been used. As the compressive load is removed (d), the folded area opens and an "ear" is created. The crack becomes longer. The subsequent load increase leads then to a new blunting and the fatigue striation created during the compression in the previous cycle is now visible (e). The following stages (f), (g), (h) are the cyclically repetition of the re-sharpening process of the previous cycle which leads to the creation of a new "ear" and thus to another striation as well as to a further increase of the crack length. The similarity to Laird's idea (the last column in Figure 2) is remarkable

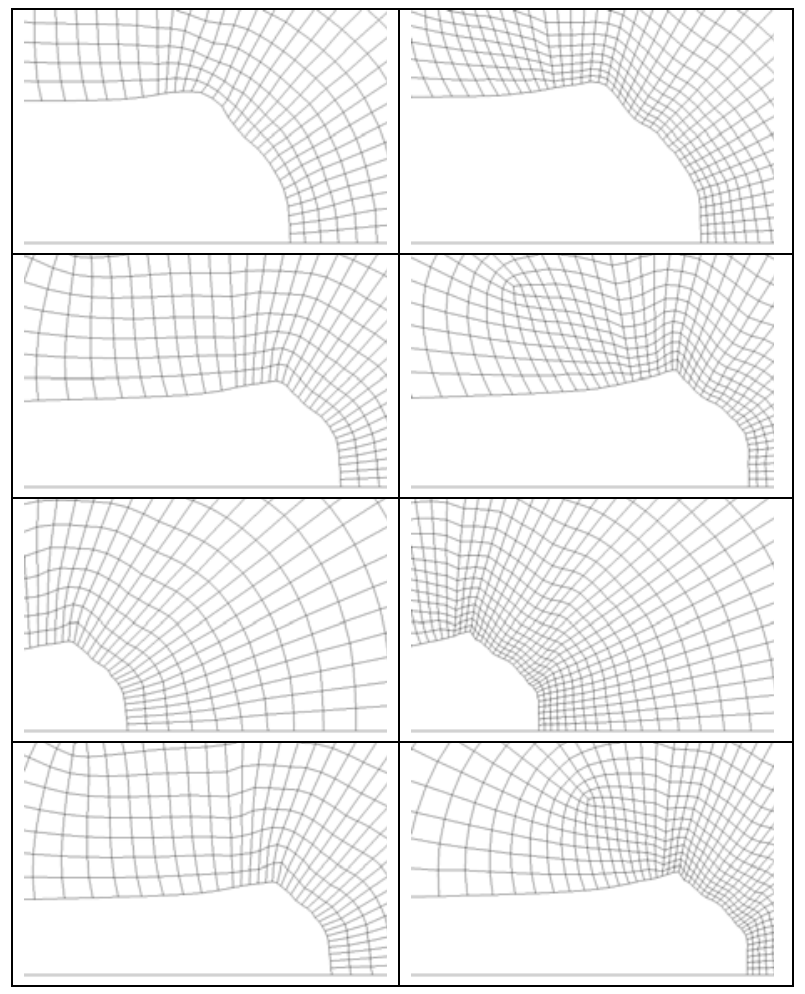

Figure 3: Simulation of crack propagation by blunting and re-sharpening for crack plane orientation (001) and crack growth direction [100]: first column - peak tensile load, second rowzero load, third row - peak compressive load, forth row - zero load.

The crack growth simulation for the crack orientation (001)[100] with the same stress level as for (001)[110] also yields crack extension by the progressive large deformations, but without striation formation. The corresponding stages are shown in Figure 3. 


\section{REFERENCES}

[1] Laird, C. and Smith, G.C. Crack propagation in high stress fatigue, Philosophical Magazine 8, 847-57, 1962.

[2] Neumann, P. New experiments concerning the slip process at propagating fatigue cracks, Acta Metallurgica 22, 1155-65, 1974.

[3] Pelloux, R.M.N. Crack extension by alternating shear, Engineering Fracture Mechanics 1, 697-704, 1970.

[4] Gu, I. and Ritchie, R.O. On the crack-tip blunting model for fatigue crack propagation in ductile materials, Fatigue and Fracture Mechanics 29, ASTM STP 1332, 552-564, 1999.

[5] Tvergaard V. and Hutchinson W. Crack growth per cycle by blunting and void growth. In: A.F. Blom (ed.), Fatigue'2002, Proc. Eighth Int. Fatigue Congress, Stockholm, Sweden, Vol. 1, EMAS, U.K., 107-116, 2002.

[6] Méric L., Poubanne P. and Cailletaud G.: Single crystal modeling for structural calculations: part 1 - model presentation. J. Engng Mat. Technology 113, 162-170, 1991. 